\title{
Cardiovascular disease and subclinical atherosclerosis in rheumatoid arthritis
}

\author{
Suad Hannawi ${ }^{1} \cdot$ Haifa Hannawi ${ }^{1}$ - Issa Al Salmi ${ }^{2}$ \\ Keywords Carotid intima-media thickness $\cdot$ rheumatoid arthritis $\cdot$ cardiovascular disease $\cdot$ atherosclerosis $\cdot$ inflammation \\ - C-reactive protein
}

Received: 19 April 2020 / Revised: 27 April 2020 / Accepted: 29 April 2020 / Published online: 1 June 2020

(c) The Japanese Society of Hypertension 2020

We read with interest the article "Traditional cardiovascular risk factors and residual disease activity are associated with atherosclerosis progression in rheumatoid arthritis patients" by Dalbeni et al. The authors assessed carotid intima-media thickness (cIMT), carotid plaques, and carotid segmental distensibility (cCD) in rheumatoid arthritis (RA) patients with no known atherosclerotic disease. In this communication, we will evaluate the cardiovascular disease (CVD) risk factors in RA and ultrasound investigations for subclinical CVD, and we will assess the study of Dalbeni et al. RA is the most common inflammatory autoimmune disease and affects $1-3 \%$ of the population worldwide. CVD is the most common cause of mortality among RA patients as a result of the combined effects of traditional and nontraditional CVD risk factors.

CVD risk factors within the RA inflammatory environment alter the structure, properties, and function of the arterial wall and impair endothelial function [1-3]. The ability to detect and monitor changes in the function and structure of arteries indicates the cumulative effect of hemodynamic, metabolic, and inflammatory stimuli in impairing arterial wall integrity and represents the potential to intervene at an early stage of RA to prevent or hinder atherosclerosis and CVD progression.

The importance of the assessment of the arterial wall has been highlighted by many studies, and they have demonstrated that arterial changes act as an independent predictor for future CVD [1, 4, 5]. Arterial changes have been examined through different noninvasive techniques, such as

Suad Hannawi

suad1@ausdoctors.net

1 Ministry of Health and Prevention, Dubai, United Arab Emirates

2 Royal Hospital, Muscat, Oman ultrasonographic assessment of cIMT, endothelial dysfunction, left anterior descending coronary artery intimamedia thickness, and many other modalities [1-3].

Traditional CVD risk factors have been found to contribute to an increased risk of CVD in RA. For instance, smoking increases CVD risk in RA, in addition to its association with more severe RA clinical presentation, radiographic damage progression, and rheumatoid factor (RF) and anticitrullinated peptide antibody (anti-CCP) positivity [6].

Dalbeni et al. evaluated the contribution of chronic inflammation and various CVD risk factors in the progression of atherosclerosis in 105 patients with RA at enrollment and after a 12-month period. They found that older age, high blood pressure, and high CRP contributed significantly to the worsening of atherosclerosis during the study period. Of note, no changes or adjustments were made in medical management, and there were no positive changes in lifestyle behaviors, such as smoking cessation. Among the RA population, $30 \%$ were smokers. In addition, the daily number of cigarettes smoked, smoking duration, and pack-year history were not available. Therefore, the percentage of smokers in the study of Dalbeni can skew the results in favor of worsened subclinical indices over a year's duration. This possibility raises a concern about the actual effect of RA alone on the subclinical atherosclerosis indices in their study. In addition, detailed RA disease activity characteristics among RA patients need to be investigated and correlated with cIMT.

Hypertension was found to be prevalent in $40 \%$ and dyslipidemia in 55-65\% of RA patients, with a significant association with CVD risk. The prevalence of insulin resistance and metabolic syndrome was reported to be increased in RA patients and was found to be related to increased CVD morbidity and mortality [7]. In a positive trend, Dalbeni et al. reported that mean arterial blood 
Table 1 Cardiovascular disease risk factors in rheumatoid arthritis

\begin{tabular}{ll}
\hline Traditional & Nontraditional \\
\hline Male gender & Inflammation \\
Age & Hyper-homocystinemia \\
Arterial hypertension & Uric acid \\
Diabetes mellitus & Subclinical renal impairment \\
Dyslipidemia & \\
Smoking & \\
Obesity & \\
Sedentary lifestyle &
\end{tabular}

Other factors had been suggested to play a role in the CVD but no enough information about its role in RA seating;1-impaired blood hemorheology (anemia, polycythemia, high white cell count parameters, thrombocytosis), 2-iron overload, 3-heamostatic changes

pressure and diabetes were independently associated with a worsening of subclinical atherosclerosis in RA patients over a year.

Despite the increase in traditional CVD risk factors among RA patients, it has been demonstrated that traditional CVD risk factors are not sufficient to explain the increase in CVD risk in RA patients. Therefore, inflammation has been included as a nontraditional CVD risk factor (Table 1). Inflammation has a key role in the onset, progression and complications of atherosclerosis [7]. Elevations in C-reactive protein (CRP) and some autoantibodies, such as RF and anti-CCP, predate the onset of RA and suggest that the accelerated atherogenic process that characterizes early RA is related to the inflammation that precedes RA symptom onset [1]. Therefore, inflammatory activity, manifested by abnormal CRP values, was considered an independent CVD risk factor in RA patients [1]. The results of the study by Dalbeni et al. are consistent with those of previous studies and revealed that persistently high CRP among RA patients was associated with atherosclerosis progression.

Therefore, different models could explain the increased risk of CVD in RA. This could be due to excess traditional CVD risk factors, such as hypertension, smoking, lipid abnormalities, hyperinsulinemia, insulin resistance, and hyperhomocysteinemia in RA patients. Another model that could explain the increase in CVD in RA patients is increased inflammation, which is the sole major driver of excess CVD. The most functional model is the integration between inflammation and traditional CVD risk factors. Inflammation has been found to be linked to traditional CVD risk factors in the RA setting. Inflammation could initiate and increase the severity of traditional CVD risk factors.

Moreover, age is a nonmodifiable risk factor for CVD. Older age at RA onset has been reported as a risk factor for excess CVD mortality. The slope of the regression line between age and cIMT has been found to be steeper in RA patients than in their controls matched for age, sex, and traditional CVD risk factors, suggesting a more rapid rate of increase in cIMT in RA patients than in controls with increasing age [1]. This can be explained by the fact that some of the traditional CVD risk factors, such as hypertension and hyperlipidemia, increase with age. On the other hand, CRP levels and inflammation severity have been found to increase with age [1]. Although age is not modifiable, many risk factors associated with age are amenable to treatment and can be managed.

In addition, while RA is more common in women, the 10-year risk of CVD was significantly higher in men than in women with RA, and male sex was positively associated with cIMT in RA patients [8]. Delbani et al. did not look at the effect of sex on the subclinical atherosclerosis indices, nor did they state the proportion of males and females among their study participants.

Nonselective and selective anti-inflammatory drugs have been reported to increase the risk of CVD. Among the nonselective anti-inflammatory drugs, the highest CVD risk was seen with diclofenac and indomethacin. Ibuprofen has been found to block the cardioprotective benefits of aspirin [9]. An imbalance between prostacyclin I2 (PGI2), a vasodilator and inhibitor of platelet aggregation, and thromboxane A2, a vasoconstrictor and promoter of platelet aggregation caused by selective cyclooxygenase inhibitors, increases the risk of CVD [10]. Although Dalbeni et al. reported NSAIDs consumption by $20 \%$ of their study participants, the effect of NSAIDs on the CVD subclinical indices has not been investigated.

Even though atherosclerotic disease may remain asymptomatic for decades, its first manifestation can be severe or even fatal. For this reason, early primary prevention of atherosclerosis and efforts to ameliorate its progression have become important goals in medicine. The noninvasive nature of B-mode ultrasonography makes it ideal for screening for the presence of subclinical CVD, monitoring its progression, and for epidemiological and longitudinal studies.

cIMT ultrasound is of particular interest because of its easy accessibility. It has been reported that ultrasonographic assessment of cIMT is an inexpensive, feasible, valid, reliable, reproducible, and cost-effective method for both clinical trials and population studies of atherosclerosis progression and regression. cIMT values measured by ultrasound correlate closely with direct measurement of local and systemic atherosclerotic burden in RA [1, 5].

A number of studies have reported a significant correlation between traditional CVD risk factors and inflammation among early and established RA patients. Of the CVD risk factors that showed an association with cIMT in a positive manner were age, body mass index, systolic blood 
pressure, diastolic blood pressure, CRP, triglyceride, lowdensity lipoprotein levels, erythrocyte sedimentation rate (ESR), hemoglobin and hematocrit levels, mean cell volume, platelet, monocyte, and eosinophil counts, ferritin, creatinine, and uric acid levels. Negative relationships were reported between cIMT and glomerular filtration rate and transferrin and high-density lipoprotein levels [5]. Additionally, it has been found that cIMT regresses with the use of multiple disease-modifying antirheumatic drugs (DMARDs) [3]. Interestingly, in the article by Dalbeni et al., of all the parameters assessed, cIMT, plaques and cCD worsened with respect to baseline measurements. This raises a question about the proper management of RA and inflammation suppression among the involved participants in the Dalbeni et al. study. Alternatively, more details of the RA disease activity and characteristics need to be investigated.

In conclusion, the analysis of the arterial changes that represent subclinical CVD provides information about the CVD risk in RA patients. The ultrasound technique allows noninvasive acquisition of the structural and functional changes of the vascular system. These changes can occur in the early stage of RA and can be influenced by age, sex, RA disease activity, inflammatory status, and traditional and nontraditional CVD risk factors. Therefore, descriptive analysis of the vascular system structural and functional changes could refine CVD risk stratification and guide therapeutic interventions.

\section{Compliance with ethical standards}

Conflict of interest The authors declare that they have no conflict of interest.

Publisher's note Springer Nature remains neutral with regard to jurisdictional claims in published maps and institutional affiliations.

\section{References}

1. Hannawi S, Haluska B, Marwick TH, Thomas R. Atherosclerotic disease is increased in recent-onset rheumatoid arthritis: a critical role for inflammation. Arthritis Res Ther. 2007;9:R116.

2. Hannawi S, Hannawi H, Alokaily F, Naredo E, Moller I, Al Salmi I. Recent-onset of rheumatoid arthritis leads to increase in wall thickness of left anterior descending coronary artery. An evidence of subclinical coronary artery disease. Saudi Med J. 2018;39:1213-7.

3. Hannawi S, Marwick TH, Thomas R. Inflammation predicts accelerated brachial arterial wall changes in patients with recentonset rheumatoid arthritis. Arthritis Res Ther. 2009;11:R51.

4. Hannawi S, AlSalmi I, Moller I, Naredo E. Uric acid is independent cardiovascular risk factor, as manifested by increased carotid intima-media thickness in rheumatoid arthritis patients. Clin Rheumatol. 2017;36:1897-902.

5. Hannawi S, Hannawi H, Alokaily F, Al Salmi I. Variables associated with subclinical atherosclerosis among rheumatoid arthritis patients of Gulf Cooperative Council countries. Saudi Med J. 2020;41:128-37.

6. Baghdadi LR, Woodman RJ, Shanahan EM, Mangoni AA. The impact of traditional cardiovascular risk factors on cardiovascular outcomes in patients with rheumatoid arthritis: a systematic review and meta-analysis. PLoS ONE. 2015;10:e0117952.

7. Jagpal A, Navarro-Millan I. Cardiovascular co-morbidity in patients with rheumatoid arthritis: a narrative review of risk factors, cardiovascular risk assessment and treatment. BMC Rheumatol. 2018;2:10.

8. Targonska-Stepniak B, Biskup M, Biskup W, Majdan M. Gender differences in cardiovascular risk profile in rheumatoid arthritis patients with low disease activity. Biomed Res Int. 2019;2019:3265847.

9. Kimmel SE, Berlin JA, Reilly M, Jaskowiak J, Kishel L, Chittams $\mathrm{J}$, et al. The effects of nonselective non-aspirin non-steroidal antiinflammatory medications on the risk of nonfatal myocardial infarction and their interaction with aspirin. J Am Coll Cardiol. 2004;43:985-90.

10. Kearney PM, Baigent C, Godwin J, Halls H, Emberson JR, Patrono C. Do selective cyclo-oxygenase-2 inhibitors and traditional non-steroidal anti-inflammatory drugs increase the risk of atherothrombosis? Meta-analysis of randomised trials. BMJ. 2006;332:1302-8. 